\title{
Some results about blow-up and global existence to a semilinear degenerate heat equation.
}

\author{
Jacques GIACOMONI
}

\begin{abstract}
In this paper, we are dealing with the following degenerate parabolic problem :

$\left(P_{t}\right)\left\{\begin{array}{l}\partial_{t} u-|x|^{2} \Delta u=g(u) \text { in } \boldsymbol{R}^{+} \times B_{1} \\ u(t, x) \equiv 0 \text { in } \boldsymbol{R}^{+} \times \partial B_{1} ; u(0, x)=u_{0} \geq 0\end{array}\right.$

where $B_{1}=\left\{x \in R^{N} ;\|x\|=1\right\}$ and $g$ is nonlinear.

We are interested in analizying such. questions as local and global existence, blow-up in finite time and convergence to a stationary solution for solutions of $\left(P_{\ell}\right)$.

First, we give some examples of nonlinearities $g$ where the blow up in $L^{2}\left(\frac{d z}{|x|^{2}}\right) \cap L^{\infty}\left(B_{1}\right)$ occurs. In a second part of this work, we present two cases of global existence of solutions to $\left(P_{t}\right)$ which converge in $L^{\infty}\left(B_{1}\right)$ to a stationary solution of $\left(P_{t}\right)$ when $t \rightarrow \infty$.
\end{abstract}

\section{Introduction}

In this work, we study the following problem :

$$
\left(P_{t}\right)\left\{\begin{array}{l}
\partial_{t} u-|x|^{2} \Delta u=g(u) \text { in } \mathbb{R}^{+} \times B_{1} \\
u(t, x) \equiv 0 \text { in } \mathbb{R}^{+} \times \partial B_{1} ; u(0, x)=u_{0} \geq 0
\end{array}\right.
$$

where $g$ is nonlinear and $B_{1}$ is the unit ball in $\mathbb{R}^{N}$.

1991 Mathematics Subject Classification: 35K65.

Servicio Publicaciones Univ. Complutense. Madrid, 1998. 
First, using Hille-Yosida theory, we prove for all $u_{0} \in L^{\infty}\left(B_{1}\right) \cap$ $L^{2}\left(\frac{d x}{\left[\left.x\right|^{2}\right.}\right)^{1}$ and $g \in W_{l o c}^{1, \infty}\left(\mathbb{R}^{N}\right)$, the local existence and the uniqueness of the solution $u(t)=S(t) u_{0}$ of $\left(P_{t}\right)$, where $S(t)$ is the semigroup associated to $\left(P_{t}\right)$. Then, we are interested in the behaviour of the solution $u(t)$ as $t$ increases. Precisely, under different assumptions of $g$ and $u_{0}$, we give on one hand, some examples of blow-up in finite time and on the other hand, some examples of global existence of solutions to $\left(P_{t}\right)$ which converge to a stationary solution of $\left(P_{t}\right)$.

Throughout this work, we keep in mind the results of [7] and [8] which deal with the stationary problem $(P)$ :

$$
(P)\left\{\begin{array}{l}
-|x|^{2} \Delta u=g(u) \text { in } B_{1} \\
u \in H_{0}^{1}\left(B_{1}\right) /\{0\} ; u \geq 0
\end{array}\right.
$$

Precisely, in [7], the authors prove the nonexistence of nontrivial solutions to $(P)$ in the case where $g$ satisfies the following assumptions:

(GS1) $\lambda-\left(\frac{N-2}{2}\right)^{2}+\lim _{s \rightarrow+\infty} \frac{g(s)}{s}>0$.

(GS2) $\forall s>0, G(s) \leq \frac{g(s) s}{2}$.

Otherwise, in [8], the authors give some results about the existence of nontrivial solutions of $(P)$ in the case where $g$ is sublinear. They treat three cases :

1. $g(u) \sim \lambda u+u^{p}-u^{q}$ where $1<p<q$

2. $g(u) \sim \lambda u-u^{p}$ where $p>1$ and $\lambda>\left(\frac{N-2}{2}\right)^{2}$

3. $g(u) \sim u^{\alpha}+\lambda u$ where $0<\alpha<1$ and $\lambda<\left(\frac{N-2}{2}\right)^{2}$

It is worth noting that in all cases, an unbounded connected branch of positive solutions in either $H_{0}^{1}\left(B_{1}\right)$ or $L^{\infty}\left(B_{1}\right)$ exists and in the second and third case, there is uniqueness of the nontrivial solution in $H_{0}^{1}\left(B_{1}\right)$. Then, it is very natural to see in which cases the nonexistence of nontrivial solutions of $(P)$ implies the blow-up in finite time for solutions of $\left(P_{t}\right)$ and when the uniqueness of the solution of $(P)$ implies the convergence to a stationary solution for solutions to $\left(P_{t}\right)$ when $t \rightarrow+\infty$. In this work, we prove some results in these directions.

So, the outline of the present paper is as follows :

$$
{ }^{1} L^{2}\left(\frac{d x}{|x|^{2}}\right):=\left\{u / \int_{B_{1}} \frac{|u|^{2}}{|x|^{2}} d x<\infty\right\}
$$


1. Local existence of solutions to $\left(P_{t}\right)$ in $\mathbb{R}^{+} \times L^{\infty} \cap L^{2}\left(\frac{d x}{|x|^{2}}\right)$.

2. Some examples of blow up in finite time for solutions to $\left(P_{t}\right)$

(a) The case $g(0)=0$

(b) The case $g(0)>0$

3. Two examples of existence of global solutions and convergence to a stationary solution.

Precisely, in Section 2, we apply Hille-Yosida theory in $L^{\infty} \cap L^{2}\left(\frac{d x}{|x|^{2}}\right)$. In Section 3, we start adapting a classical spectral method (see for instance. [4]) to prove the blow-up in finite time when $g$ satisfies :

(B1) $g$ is convex and positive in $\boldsymbol{R}^{+}$.

(B2) $\left(\frac{N-2}{2}\right)^{2}<\lim _{s \rightarrow 0^{+}} \frac{g(s)}{s}=\lambda<+\infty$.

(B3) There exists $s_{0}>0$ such that $\int_{s_{0}}^{+\infty} \frac{d s}{g(s)-\lambda s}<+\infty$

Next, we use a well known "energy method" (see for instance [4]). For this, we assume the following hypothesis :

(B4) $\lambda=\lim _{s \rightarrow 0^{+}} \frac{g(s)}{s}<+\infty$ and there exists $\alpha>0, C>0$ such that $h(s)=g(s)-\lambda s \geq C s^{\alpha+1}$, for all $s \geq 0$.

(B5) There exists $\epsilon>0$ such that $(2+\epsilon) \int_{0}^{s} h(t) d t \leq s h(s), \forall s>0$.

Then, we prove that if $u_{0}$ satisfies $\int_{B_{1}} \frac{\left|\nabla u_{0}\right|^{2}}{2}-\int_{B_{1}} \frac{G\left(u_{0}\right)}{|x|^{2}}<0$, where $G(s)=\int_{0}^{s} g(t) d t$, the solution $u(t)$ to $\left(\bar{P}_{t}\right)$ blows up in finite time. Finally, we conclude the section with the case $g(0)>0$. Precisely, we apply a method from [3] which links directly the blow-up and the nonexistence of stationary solutions. For this, we assume :

(B6) $g(0)>0, g \in C^{1}([0,+\infty[)$, convex and increasing.

(B7) There exists $x_{0}>0$ such that $\int_{x_{0}}^{+\infty} \frac{d s}{g(s)}<\infty$. 
Then, for any $u_{0} \geq 0$, the solution $u(t)=S(t) u_{0}$ blows up in finite time.

In Section 4, we give some results concerning the existence of global solutions to $\left(P_{t}\right)$. First, proving the radial symmetry of the solution to $\left(P_{t}\right)$ when $u_{0}$ is radially symmetric, we exhibit the heat kernel of $-|x|^{2} \Delta$ in $H_{0}^{1}\left(B_{1}\right)$. Then, using a method due to Fujita, we prove the existence of a global solution of $\left(P_{t}\right)$ for small initial data when $g(t) \sim \lambda t+t^{p}$, $p>1$ and $\lambda<0$. Moreover, we prove that $u(t)$ converges to 0 in $L^{\infty}\left(B_{1}\right)$ with an exponentional decay when $t \rightarrow+\infty$.

Finally, assuming the following hypothesis :

(G3) $s \rightarrow \frac{g(s)}{s}$ is continuous and strictly decreasing,

(G4) $\stackrel{g(s)}{s} \stackrel{s \rightarrow+\infty}{\longrightarrow}-\infty$,

(G5) $\lim _{s \rightarrow 0^{+}} \frac{g(s)}{s}=\lambda>\left(\frac{N-2}{2}\right)^{2}$,

we show that for any $u_{0}>0$ satisfying $u_{0} \in L^{\infty} \cap L^{2}\left(\frac{d x}{|x|^{2}}\right),\left\|u_{0}\right\|_{L^{\infty}} \leq$ $f^{-1}(0)$ and $u_{0} \not \equiv f^{-1}(0)$ where $f(t):=\frac{g(t)}{t}$, the solution $u(t)$ of $\left(P_{t}\right)$ is global and converges to the unique nontrivial stationary solution of $\left(P_{t}\right)$ in $L^{\infty}\left(B_{1}\right) \cap H_{0}^{1}\left(B_{1}\right)$.

\section{Local existence}

Throughout this section, we assume that $g \in W_{l o c}^{1, \infty}(\mathbb{R})$. Our goal is to study the local existence of a solution to $\left(P_{t}\right)$. Precisely, we show that we can apply Hille-Yosida theory in $L^{\infty}\left(B_{1}\right) \cap L^{2}\left(\frac{d x}{\mid \sqrt{||^{2}}}\right)$. Consequently, for every $u_{0} \in L^{\infty} \cap L^{2}\left(\frac{d x}{|x|^{2}}\right)$, the uniqueness of solutions of $\left(P_{t}\right)$ follows. First, we remark :

Proposition 2.1. Let $A=-|x|^{2} \Delta$. Then, $A$ is a self adjoint maximal monotone operator in $L^{2}\left(\frac{d x}{|x|^{2}}\right)$. Moreover, $\mathcal{D}(A)=\left\{u \in L^{2}\left(\frac{d x}{|x|^{2}}\right) / u \in\right.$ $H_{0}^{1}\left(B_{1}\right)$ and $\left.|x|^{2} \Delta u \in L^{2}\left(\frac{d x}{|x|^{2}}\right)\right\}$.

Proof. For this, notice that for every $u \in \mathcal{D}(A)$ and $\lambda>0$ :

$$
\begin{aligned}
\left\|u-\lambda|x|^{2} \Delta u\right\|_{L^{2}\left(\frac{d x}{|x|^{2}}\right)}^{2} & =\|u\|_{L^{2}\left(\frac{d x}{|x|^{2}}\right)}^{2}+2 \lambda\|\nabla u\|_{L^{2}}^{2} \\
& +|\lambda|^{2} \int_{B_{1}}|x|^{2}|\Delta u|^{2} \geq\|u\|_{L^{2}\left(\frac{d x}{|x|^{2}}\right)}^{2}
\end{aligned}
$$


which implies that $A$ is dissipative in $L^{2}\left(\frac{d x}{|x|^{2}}\right)$. Then, it suffices to show that $A$ is maximal. Taking $f \in L^{2}\left(\frac{d x}{|x|^{2}}\right)$, we consider the following minimization problem :

$$
\begin{aligned}
& I_{\lambda}=\inf _{u \in H_{0}^{1}\left(B_{1}\right)} \mathcal{E}(u) \\
& \text { where } \quad \mathcal{E}(u)=\frac{1}{2} \int_{B_{1}}\left(\frac{|u|^{2}}{|x|^{2}}+\lambda|\nabla u|^{2}\right) d x-\int_{B_{1}} \frac{f u}{|x|^{2}}
\end{aligned}
$$

By Cauchy-Schwarz's inequality,

$$
I_{\lambda} \geq \inf _{u \in I_{0}^{1}\left(B_{1}\right)} \frac{1}{2} \int_{B_{1}}\left(\frac{|u|^{2}}{|x|^{2}}+\lambda|\nabla u|^{2}\right) d x-\left(\int_{B_{1}} \frac{|f|^{2}}{|x|^{2}}\right)^{\frac{1}{2}}\left(\int_{B_{1}} \frac{|u|^{2}}{|x|^{2}}\right)^{\frac{1}{2}}>-\infty
$$

then, considering a minimizing sequence $\left\{u_{n}\right\}_{n \in N} \subset H_{0}^{1}\left(B_{1}\right) \cap L^{2}\left(\frac{d x}{|x|^{2}}\right)$, it follows that $\left\|u_{n}\right\|_{H_{0}^{1} \cap L^{2}\left(\frac{d x}{|x|^{2}}\right)} \leq C$. And by standard compactness arguments, there exists $u \in H_{0}^{1}\left(B_{1}\right) \cap L^{2}\left(\frac{d x}{|x|^{2}}\right)$ such that up to subsequences :

$$
u_{n} \underset{n \rightarrow \infty}{\rightarrow} u \text { weakly in } H_{0}^{1}\left(B_{1}\right), u_{n} \underset{n \rightarrow \infty}{\rightarrow} u \text { weakly in } L^{2}\left(\frac{d x}{|x|^{2}}\right)
$$

and

$$
\int_{B_{1}} \frac{f u_{n}}{|x|^{2}} \stackrel{n \rightarrow \infty}{\longrightarrow} \int_{B_{1}} \frac{f u}{|x|^{2}}
$$

Therefore, $I_{\lambda}$ is achieved by $u$ and the proof is complete.

We deduce immediatly the following corollary :

Corollary 2.2. $A$ is maximal monotone in $L^{\infty}\left(B_{1}\right) \cap L^{2}\left(\frac{d x}{|x|^{2}}\right)$. Moreover, $\mathcal{D}(A)=\left\{u \in H_{0}^{1}\left(B_{1}\right) \cap L^{\infty} /|x|^{2} \Delta u \in L^{2}\left(\frac{d x}{|x|^{2}}\right) \cap L^{\infty}\right\}$.

Proof. Let $f \in L^{2}\left(\frac{d x}{|x|^{2}}\right) \cap L^{\infty}$ and $\lambda>0$. By Proposition 2.1, there exists $u \in H_{0}^{1} \cap L^{2}\left(\frac{d x}{+x^{2}}\right)$ such that

$$
u-\lambda|x|^{2} \Delta u=f \text { in } B_{\mathrm{I}}
$$


Thus, it suffices to show that $u \in l^{\infty}\left(B_{1}\right)$. Multiplying (2.1) by $\left(u-\|f\|_{L^{\infty}}\right)^{+}$, we obtain :

$$
\begin{aligned}
& \int_{B_{1}} \frac{\frac{\left(u-\|f\|_{L^{\infty}}\right)^{+2}}{|x|^{2}} d x+\lambda \int_{B_{1}} \mid \nabla\left(u-\|f\|_{L^{\infty}}\right)^{+}}{=}=0 \\
& =\int_{B_{1}}\left(f-\|f\|_{L^{\infty}}\right) \frac{\left(u-\|f\|_{L^{\infty}}\right)^{+}}{|x|^{2}} \leq 0
\end{aligned}
$$

which yields $\left(u-\|f\|_{L^{\infty}}\right)^{+} \equiv 0$ and $u \leq\|f\|_{L^{\infty}}^{-}$. By the same arguments, we show that $u \geq-\|f\|_{L^{\infty}}$. This ends the proof of Corollary 2.2 .

Remark. For $N \geq 3, L^{\infty}\left(B_{1}\right) \subset L^{2}\left(\frac{d x}{|x|^{2}}\right)$. And in this case, to prove Corollary 2.2, it suffices to show the maximality of $A$ in $L^{\infty}$.

Now, we apply Hille-Yosida theory (see [15]) and we deduce the following proposition :

Proposition 2.3. Let $u_{0} \in L^{\infty}\left(B_{1}\right) \cap L^{2}\left(\frac{d x}{|x|^{2}}\right)$. Then, there exists a unique solution $u(t)=S(t) u_{0}$ to $\left(P_{t}\right)$ in a maximal interval $[0, T[, T>0$ such that

(i) $u(\cdot) \in C^{0}\left(\left[0, T\left[, L^{\infty}\left(B_{1}\right) \cap L^{2}\left(\frac{d x}{|x|^{2}}\right)\right) \cap C^{1}(] 0, T\left[, L^{2}\left(\frac{d x}{|x|^{2}}\right)\right)\right.\right.$.

(ii) For allt in $] 0, T\left[, u(t) \in H_{0}^{1}\left(B_{1}\right) \cap L^{\infty} \cap L^{2}\left(\frac{d x}{|x|^{2}}\right)\right.$ and $|x|^{2} \Delta u(t) \in$ $L^{2}\left(\frac{d x}{|x|^{2}}\right)$.

(iii) If $u_{0} \geq 0$, then $u(t) \geq 0$ for all $t>0$.

(iv) If $u_{0} \in L^{\infty}\left(B_{1}\right) \cap L^{2}\left(\frac{d x}{|x|^{2}}\right)$ satisfies $|x|^{2} \Delta u_{0} \in L^{\infty}\left(B_{1}\right) \cap L^{2}\left(\frac{d x}{|x|^{2}}\right)$, then $u(t) \in C^{1}\left(\left[0, T\left[, L^{\infty}\left(B_{1}\right) \cap L^{2}\left(\frac{d x}{|x|^{2}}\right)\right.\right.\right.$.

Proof. By Proposition 2.1, Corollary 2.2 and since $g \in W_{\text {loc }}^{1, \infty}$ we can apply Theorems 3.7 and 3.9 of [4]. This proves assertions (i), (ii) and (iv). Now, let us prove assertion (iii). For every $T_{0}<T$, we multiply the equation in $\left(P_{t}\right)$ by $\frac{(-u)^{-}}{|x|^{2}}$ and integrate by parts to obtain for every $t \in\left[0, T_{0}\right]:$

$$
\frac{1}{2} \frac{d}{d t} \int_{B_{1}} \frac{\left|u^{-}\right|^{2}}{|x|^{2}} d x=-\int_{B_{1}}\left|\nabla u^{-}\right|^{2}-\int_{B_{1}} \frac{g(u) u^{-}}{|x|^{2}} \leq C\left(T_{0}\right) \int_{B_{1}} \frac{\left|u^{-}\right|^{2}}{|x|^{2}}
$$


which implies by Gronwall's lemma that $u^{-} \equiv 0$. This completes the proof of Proposition 2.3.

As a consequence of Hille-Yosida Theory, we have the following alternative for $u(t)=S(t) u_{0}$ :

Corollary 2.4. If $u_{0} \in L^{2}\left(\frac{d x}{|x|^{2}}\right) \cap L^{\infty}$, then, either $T=T\left(\left\|u_{0}\right\|_{\mathcal{D}(A)}\right)=$ $+\infty$ and the solution $u(\cdot)=S(\cdot) u_{0}$ is global, or $T<+\infty$ and the solution blows up in finite time which means that

$$
\|u(t)\|_{L^{\infty}}+\|u(t)\|_{L^{2}\left\{\frac{d x}{|x|^{2}}\right)} \stackrel{t \rightarrow T^{-}}{\longrightarrow}+\infty
$$

Proof. See [4].

Remarks. If $g \equiv 0$ and $u_{0} \in H^{1} \cap L^{\infty} \cap L^{2}\left(\frac{d x}{|x|^{2}}\right)$ then $u(t)=S(t) u_{0}$ is global and satisfies:

$$
\int_{B_{1}} \frac{|u(t)|^{2}}{|x|^{2}} \leq e^{-\left(\frac{N-2}{2}\right)^{2} t}|| u_{0} \|_{L^{2}\left(\frac{d x}{\mid\left(\frac{1 T^{2}}{2}\right)}\right.}^{2}
$$

The proof is based upon Hardy's inequality. First, observe that since $g \equiv 0,\left(P_{t}\right)$ is linear. Therefore, $u(t)=S(t) u_{0}$ is global. Moreover, multiplying $\left(P_{t}\right)$ by $u(t) e^{\left(\frac{N-2}{2}\right)^{2} t}$ and integrating by parts, we have :

$$
\begin{aligned}
& \frac{d}{d t} \int_{B_{1}} \frac{\left|u^{-}\right|^{2}}{|x|^{2}} e^{\left(\frac{N-2}{2}\right)^{2} t} d x=2\left(\frac{N-2}{2}\right)^{2} \int_{B_{1}} \frac{\left|u^{-}\right|^{2}}{|x|^{2}} e^{\left(\frac{N-2}{2}\right)^{2} t} d x-2 \\
& \int_{B_{1}}|\nabla u|^{2} e^{\left(\frac{N-2}{2}\right)^{2} t} d x \leq 0
\end{aligned}
$$

by Hardy's inequality. Thus, integrating on $[0, t]$, we deduce (2.2).

Now, we deal with the behaviour of the solution to $\left(P_{t}\right)$. In the next section, we give some examples of blow-up in finite time of solutions to $\left(P_{t}\right)$.

\section{Blow up in finite time in $L^{2}\left(\frac{d x}{|x|^{2}}\right) \cap L^{\infty}$}

Throughout this section, we assume that $g$ belongs to $W_{l o c}^{1, \infty}, u_{0} \in L^{\infty} \cap$ $L^{2}\left(\frac{d x}{|x|^{2}}\right)$ and $G(s)=\int_{0}^{s} g(t) d t$. 


\subsection{Main results}

We consider three classes of functions $g$. First, we adapt a classical "spectral method" (see for instance [4]). Precisely, we prove the following theorem :

Theorem 3.1.Assuming $N \geq 3$ and

(B1) $g$ is convex and positive in $\mathbb{R}^{+}$,

(B2) $\left(\frac{N-2}{2}\right)^{2}<\lambda:=\lim _{s \rightarrow 0^{+}} \frac{g(s)}{s}<+\infty$,

(B.3) There exists $s_{0}>0$ such that $\int_{s_{0}}^{+\infty} \frac{d s}{h(t)}<\infty$ where $h(s)=g(s)-$ $\lambda s$.

Then, for any $u_{0} \geq 0$ in $L^{\infty} \cap L^{2}\left(\frac{d x}{|x|^{2}}\right), u(t)=S(t) u_{0}$ satisfies : $\exists T \in$ $\mathbb{R}^{+}$such that

$$
\lim _{t \rightarrow T^{-}} \int_{B_{1}} \frac{|u(t)|^{2}}{|x|^{2}}=+\infty \text { and } \lim _{t \rightarrow T^{-}}\|u(t)\|_{L^{\infty}}=+\infty
$$

The second blow-up case is based upon an "energy method" (see for instance [4]).

Theorem 3.2. Assume that $u_{0}$ satisfies $\left(^{*}\right) \int_{B_{1}} \frac{\left|\nabla u_{0}\right|^{2}}{2}-\int_{B_{1}} \frac{G\left(u_{0}\right)}{|x|^{2}}<0$ and that $g$ has the following properties:

(B4) $\lambda:=\lim _{s \rightarrow 0^{+}} \frac{g(s)}{-s^{-}} \in \mathbb{R}$ and there exists $\alpha>0, C>0$ such that $h(s)=g(s)-\lambda s \geq C s^{\alpha+1}$ for all $s \geq 0$,

(B5) There exists $\epsilon>0$ such that for all $s \geq 0,(2+\epsilon) H(s) \leq \operatorname{sh}(s)$ where $H(t)=\int_{0}^{t} h(s) d s$.

Then, $u(t)=S(t) u_{0}$ satisfies : $\exists T>0$ such that $\lim _{t \rightarrow T^{-}} \int_{B_{1}} \frac{|u(t)|^{2}}{|x|^{2}}=$ $+\infty$.

\section{Remarks.}

1. If $g(s)=\lambda s+s^{p}$ with $\lambda>\left(\frac{N-2}{2}\right)^{2}$ and $p>1,(\mathrm{~B} 1)$, (B2) and (B3) are satisfied. 
2. If $g(s)=\lambda s+s^{p}$ with $p>1,(\mathrm{~B} 4)$ and (B5) are satisfied.

3. Let $\phi \in L^{\infty} \cap H_{0}^{1}$. Then, by (B4), there exists $M>0$, large enough, such that $u_{0}=M \phi$ satisfies $\left({ }^{*}\right)$.

4. If $u_{0} \geq 0$ is a radially decreasing nontrivial subsolution of $(P)$ and belongs to $H_{0}^{1}\left(B_{1}\right) \cap L^{\infty}$, then, a simple computation based upon a "Pohozaev's equality type" shows that $\left(^{*}\right)$ is satisfied for $N>2$. Indeed, multiplying $-|x|^{2} \Delta u_{0} \leq g\left(u_{0}\right)$ by $\frac{x}{|x|^{2}} \cdot \nabla u_{0}$ and integrating by parts, we obtain :

$$
-\left(\frac{N-2}{2}\right) \int_{B_{1}}\left|\nabla u_{0}\right|^{2}-\frac{1}{2} \int_{\partial B_{1}}\left|\frac{\partial u_{0}}{\partial n}\right|^{2} d s \geq(2-N) \int_{B_{1}} \underline{\frac{G\left(u_{0}\right)}{|x|^{2}}}
$$

which implies:

$$
\int_{B_{1}} \frac{\left|\nabla u_{0}\right|^{2}}{2}-\int_{B_{1}} \frac{G\left(\underline{\left.u_{0}\right)}\right.}{|x|^{2}} \leq-\frac{1}{2(N-2)} \int_{\partial B_{1}}\left|\frac{\partial u_{0}}{\partial n}\right|^{2} d s<0
$$

Finally, we deal with the case $g(0)>0$. In this case, we adapt a method from [3]. And we use the results of nonexistence of solutions to the problem $(\mathrm{P})$.

Theorem 3.3. Assume that $N \geq 3$ and the following assumptions on $g$ :

(B6) $g>0$ is convex, increasing and belongs to $C^{1}([0,+\infty[)$,

(B7) There exists $s_{0}>0$ such that $\int_{s_{0}}^{+\infty} \frac{d s}{g(s)}<\infty$.

Then, for all $u_{0} \geq 0$ in $L^{\infty} \cap L^{2}\left(\frac{d x}{|x|^{2}}\right)$ and nontrivial, $u(t)=S(t) u_{0}$ blows up in finite time in $L^{\infty}$ and in $L^{2}\left(\frac{d x}{|x|^{2}}\right)$.

\section{Remarks.}

1. It is worth noting that in Theorems 3.1 and 3.3 , no additional assumption is required for $u_{0}$. Here, the nonexistence of weak nontrivial solutions of the stationary problem $(P)$ implies the blowup in finite time for any initial data in $L^{\infty} \cap L^{2}\left(\frac{d x}{|x|^{2}}\right)$.

2. The assumptions (B3) and (B7) prevent the existence of unbounded global solutions (i.e. which blow up when $t \rightarrow \infty$ ). 
Now, we prove Theorem 3.1:

Proof of Theorem 3.1. Let us consider $\psi_{\epsilon}$ the eigenfunction associated with the first eigenvalue $\lambda_{\varepsilon}^{1}$ of $-\left(|x|^{2}+|\epsilon|^{2}\right) \Delta$ in $H_{0}^{1}\left(B_{1}\right)$ such that $\int_{B_{1}} \frac{\psi_{c}}{|x|^{2}}=1$ (for this, notice that $N \geq 3$ implies that $L^{2}\left(\frac{d x}{|x|^{2}}\right) \subset L^{\mathbf{1}}\left(\frac{d x}{|x|^{2}}\right)$ ). It is easy to prove that $\lambda_{\epsilon}^{1} \longrightarrow\left(\frac{N-2}{2}\right)^{2}$ when $\epsilon \rightarrow 0$. Therefore, by (B2), there exists $\epsilon>0$ small enough such that $\lambda_{\epsilon}^{1}<\lambda$. Thus, multiplying $\left(P_{t}\right)$ by $\frac{\psi_{c}}{|x|^{2}}$, we obtain :

$$
\frac{d}{d t} \int_{B_{1}} \frac{u(t) \psi_{\epsilon}}{|x|^{2}}+\lambda_{\epsilon}^{\mathrm{t}} \int_{B_{1}} \frac{u(t)}{|x|^{2}+|\epsilon|^{2}}=\int_{B_{1}} \frac{g(u(t)) \psi_{\epsilon}}{|x|^{2}}
$$

Since $g$ is convex (which implies that $f$ is convex), by Jensen's inequality, we have :

$$
\frac{d}{d t} \int_{B_{1}} \frac{u(t) \psi_{\epsilon}}{|x|^{2}} \geq\left(\lambda-\lambda_{\varepsilon}^{1}\right) \int_{B_{1}} \frac{u(t) \psi_{\epsilon}}{|x|^{2}}+h\left(\int_{B_{1}} \frac{u(t) \psi_{\epsilon}}{|x|^{2}}\right)
$$

From which it follows :

$$
\frac{d}{d t}\left(\int_{0}^{\phi(t)} \frac{d s}{h(s)}\right) \geq 1 \quad \text { where } \phi(t)=\int_{B_{1}} \frac{\left.x\right|^{2}}{\mid t(t)}
$$

Integrating (3.1), one has $\int_{0}^{\phi(t)} \frac{d s}{h(s)} \geq t+C$ which together with (B3) implies that $\phi(\cdot)$ blows up in finite time. Finally, noting that for $N \geq 3$, the injection $L^{\infty} \hookrightarrow L^{2}\left(\frac{d x}{|x|^{2}}\right)$ is continuous, the proof of Theorem 3.1 is complete.

Next, we give the proof of Theorem 3.2:

Proof of Theorem 3.2. Suppose that the solution $u(t)=S(t) u_{0}$ is global. Let us consider $E(t)=\frac{1}{2} \int_{B_{1}}|\nabla u(t)|^{2}-\int_{B_{1}} \frac{G(u(t))}{|x|^{2}}$. Then, multiplying $\left(P_{t}\right)$ by $\frac{u_{t}}{\mid x^{2}}$ and integrating by parts, we obtain :

$$
\int_{B_{1}} \frac{\left|u_{t}\right|^{2}}{|x|^{2}}=-\frac{1}{2} \frac{d}{d t} \int_{B_{1}}|\nabla u(t)|^{2}+\frac{d}{d t} \int_{B_{1}} \frac{G(u(t))}{|x|^{2}}=-\frac{d}{d t}(E(t))
$$

Thus, $E(t)$ is decreasing and $E(t) \leq E(0)<0$. Now, multiplying the equation in $\left(P_{t}\right)$ by $\frac{u(t)}{|x|^{2}}$ and integrating by parts :

$$
\frac{1}{2} \frac{d}{d t} \int_{B_{1}} \frac{|u(t)|^{2}}{|x|^{2}}=-\int_{B_{1}}|\nabla u(t)|^{2}+\int_{B_{1}} \frac{g(u(t)) u(t)}{|x|^{2}}
$$


By using (B5), and taking $H(s)=\int_{0}^{s} h(t) d t$, we prove that :

$$
\begin{aligned}
\frac{1}{2} \frac{d}{d t} \int_{B_{1}} \frac{|u(t)|^{2}}{|x|^{2}} & \geq-\int_{B_{1}}|\nabla u(t)|^{2}+(2+\epsilon) \int_{B_{1}} \frac{H(u(t))}{|\bar{x}|^{2}}+\lambda \int_{B_{1}} \frac{|u(t)|^{2}}{|x|^{2}} \\
& \geq-2 E(t)+\epsilon \int_{B_{1}} \frac{H(u(t))}{|x|^{2}} \\
& \geq-2 E(0)+\epsilon \int_{B_{1}} \frac{H(u(t))}{|x|^{2}}
\end{aligned}
$$

Thus, (3.3) and $\left(^{*}\right)$ imply that $\lim _{t \rightarrow \infty} \int_{B_{1}} \frac{|u(t)|^{2}}{|x|^{2}}=+\infty$. Then, by (3.3) : $\frac{1}{2} \frac{d}{d t} \int_{B_{1}} \frac{|u(t)|^{2}}{|x|^{2}} \geq \epsilon \int_{B_{1}} \frac{H(u(t))}{|x|^{2}} \geq \epsilon \epsilon \int_{B_{1}} \frac{|u(t)|^{2+\alpha}}{|x|^{2}} \geq C \epsilon\left(\int_{B_{1}} \frac{|u(t)|^{2}}{|x|^{2}}\right)^{\frac{3+\alpha}{3}}$

Taking $\phi(t)=\int_{B_{1}} \frac{|u(t)|^{2}}{|x|^{2}}$, we have :

$$
\frac{d}{d t} \phi(t) \geq 2 \epsilon C \phi(t)^{\frac{\alpha+2}{2}}
$$

Integrating (3.4) on $\left[t_{0}, t\right]$, we obtain :

$$
\frac{1}{\phi(t)^{\frac{\alpha}{2}}}-\frac{1}{\phi\left(t_{0}\right)^{\frac{\alpha}{2}}} \geq C\left(t-t_{0}\right)
$$

which contradicts that $u(\cdot)$ is a global solution of $\left(P_{t}\right)$. This completes the proof of Theorem 3.2 .

Finally, we prove Theorem 3.3. Here, we use an approach from [3] : the nonexistence of stationary weak solutions implies the nonexistence of global, bounded solution of $\left(P_{t}\right)$ for every $u_{0} \geq 0$.

First, we adapt the definition of a weak stationary solution of $\left(P_{t}\right)$ from [3] :

Definition 3.1. A weak stationary solution of $\left(P_{t}\right)$ is a funclion $u \in$ $L^{1}\left(B_{1}\right)$ such that $\frac{g(u)}{|x|^{2}} \delta(x) \in L^{1}\left(B_{1}\right)$ (where $\left.\delta(x)=\operatorname{dist}\left(x, \partial B_{1}\right)\right)$ and

$$
\forall \xi \in C^{2}\left(\bar{B}_{1}\right)-\int_{B_{1}} u \Delta \xi d x=\int_{B_{1}} \frac{g(u)}{|x|^{2}} \xi d x
$$


Then, we have the following result :

Proposition 3.4. Assume that $g$ satisfies (B6) and (B7). Then, there is no weak stationary solution of $\left(P_{t}\right)$. Proof .

We apply a method from [3]. Precisely, for all $\eta$ such that $0 \leq \eta<1$, we define :

$$
\left(P_{\eta}\right)\left\{\begin{aligned}
-|x|^{2} \Delta u & =(1-\eta) g(u) \text { in } B_{1} \\
u & \geq 0, \quad u \in \mathrm{H}_{0}^{1}\left(B_{1}\right)
\end{aligned}\right.
$$

As in [3], we define $h(u)=\int_{0}^{u} \frac{d s}{g(t)}, \grave{h}(u)=\frac{1}{1-\eta} h(u)$ and $\Phi(u)=\tilde{h}^{-1}(h(u))$. It is easy to prove the following assertions (see [3]) :

(i) $\Phi(0)=0$ and $0 \leq \Phi(u) \leq u$.

(ii) $\Phi$ is increasing and concave. Moreover, $\Phi^{\prime}(u) \leq 1$.

(iii) $\Phi \in L^{\infty}$ and $\Phi(u)$ satisfies :

$$
\forall \xi \in C^{2}\left(B_{1}\right)-\int_{B_{1}}(\Delta \Phi(u)) \xi \geq(1-\eta) \int_{B_{1}} \frac{g(\Phi(u)) \xi}{|x|^{2}}
$$

which means that $\Phi(u)$ is a "weak supersolution" of $\left(P_{\eta}\right)$.

Forall $\xi \in C_{0}^{2}\left(\bar{B}_{1}\right)$, let us consider the following iterative scheme :

$$
\left\{\begin{aligned}
-\int_{B_{1}} u_{n+1} \Delta \xi & =(1-\eta) \int_{B_{1}} \frac{g\left(u_{n}\right) \xi}{|x|^{2}} \text { in } B_{1} \\
u_{0} & =\Phi(u), \quad u \in \mathrm{H}_{0}^{1}\left(B_{1}\right)
\end{aligned}\right.
$$

Then, noting that $\Phi(u) \in L^{\infty}$ implies that for $N \geq 3, \frac{q(\Phi(u))}{|x|^{2}} \in L^{1}$ and by the fact that 0 is a strict subsolution to $\left(P_{\eta}\right)$, we prove, by the maximun principle, that in $L^{\infty},\left\{u_{n}\right\}_{n \geq 1}$ is a decreasing sequence of weak supersolutions of $\left(P_{\eta}\right)$ and $u_{n} \leq \boldsymbol{\Phi}(u)$. Thus, $v_{\eta}=\lim _{n \rightarrow \infty} u_{n} \in L^{\infty}$ is a weak solution of $\left(P_{\eta}\right)$. Now, consider for all $\epsilon$ in $] 0,1[$, the following problem :

$$
\left(P_{\epsilon, \eta}\right)\left\{\begin{aligned}
-\left(|x|^{2}+|\epsilon|^{2}\right) \Delta v & =(1-\eta) g(v) \text { in } B_{1} \\
v & \geq 0, v \in \mathrm{H}_{0}^{1}\left(B_{1}\right)
\end{aligned}\right.
$$

As in [1], we prove the existence of a minimal solution of $\left(P_{\varepsilon, \eta}\right), v_{\varepsilon, \eta}$, such that $v_{\varepsilon, \eta} \leq v_{\eta} \leq \Phi(u)$. 
Putting $w_{\epsilon, \eta}(x):=v_{\epsilon, \eta}(\epsilon x)$, for $x \in B_{\frac{1}{\epsilon}}$, we have :

$$
\left\{\begin{aligned}
-\left(|x|^{2}+1\right) \Delta w_{\epsilon, \eta} & =(1-\eta) g\left(w_{\epsilon, \eta}\right) \text { in } B_{\frac{1}{\epsilon}} \\
w_{\epsilon, \eta} & \geq 0, \quad w_{\epsilon, \eta} \in \mathrm{H}_{0}^{1}\left(B_{\frac{1}{\epsilon}}\right)
\end{aligned}\right.
$$

As above, we can show that $\epsilon \longrightarrow w_{\varepsilon, \eta}$ is increasing in $L^{\infty}$. Passing to the limit $\epsilon \rightarrow 0$, it is easy to prove that $w:=\lim _{\epsilon \rightarrow 0} w_{\varepsilon}$ satisfies $\|w\|_{L^{\infty}} \leq$ $\left\|v_{\eta}\right\|_{L^{\infty}}$ and is the minimal non trivial solution of the following problem :

$$
\left\{\begin{aligned}
-\left(|x|^{2}+1\right) \Delta w & =(1-\eta) g(w) \text { in } \mathbb{R}^{N} \\
w & \geq 0
\end{aligned}\right.
$$

Therefore, $w(x)=\frac{1}{C_{N}|x|^{N-2}} * \frac{g(w)}{|x|^{2}+1} \quad$ where $C_{N}=(N-2)\left|\sigma_{N-1}\right|$ and $\left|\sigma_{N-1}\right|$ the surface area of the unit sphere. Thus,

$$
\begin{aligned}
w(0)= & \int_{\mathbb{R}^{N}} \frac{C_{N} g(w)}{|x|^{N-2}\left(|x|^{2}+1\right)} d x \geq \inf _{s \in\left[0,\|w\|_{\left.L_{\infty}\right]}\right.} g(s) \\
& \int_{\mathbb{R}^{N}} \frac{1}{\left(|x|^{2}+1\right)|x|^{N-2}}=+\infty
\end{aligned}
$$

This contradicts the boundedness of $w$ and the proof of Proposition 3.4 is now complete.

Proof of Theorem 3.3. First, note that by the maximum principle, it suffices to prove Theorem 3.3 in the case $u_{0} \equiv 0$ (note that since $g$ is increasing, $\left.u_{0} \leq w_{0} \Rightarrow \forall t \geq 0, S(t) u_{0} \leq S(t) w_{0}\right)$. Moreover, $g(0)>0 \Rightarrow u_{t}>0$ for $t$ small. Then, for $\delta>0$ small,

$u(t+\delta)=S(t+\delta) 0=S(t) \circ S(\delta) 0 \geq S(t) 0=u(t)$ and $u_{t} \geq 0, \forall t \geq 0$

Now, taking $\phi \in C_{0}^{2}\left(\bar{B}_{1}\right)$, multiplying the equation in $\left(P_{t}\right)$ by $\frac{\phi}{|x|^{2}}$ and integrating by parts, we obtain :

$$
\frac{d}{d t} \int_{B_{1}} \frac{u(t) \phi}{|x|^{2}}-\int_{B_{1}} u \Delta \phi=\int_{B_{1}} \frac{g(u(t)) \phi}{|x|^{2}}
$$


Therefore, choosing $\phi=\psi_{\epsilon}$ (defined in the proof of Theorem 3.1) we have:

$$
\frac{d}{d t} \int_{B_{1}} \frac{u(t) \psi_{\varepsilon}}{|x|^{2}}+\lambda_{\epsilon}^{1} \int_{B_{1}} \frac{u \psi_{\varepsilon}}{|x|^{2}+|\epsilon|^{2}}=\int_{B_{1}} \frac{g(u(t)) \psi_{\epsilon}}{|x|^{2}}
$$

Thus,

$$
\frac{d}{d t} \int_{B_{1}} \frac{\underline{u(t) \underline{\psi}_{t}}}{|x|^{2}} \geq \int_{B_{1}}\left(\frac{g(u(t))}{u(t)}-\lambda_{\epsilon}^{1}\right) \frac{u(t) \psi_{\epsilon}}{|x|^{2}}
$$

which provides the following alternative :

1. either there exists $M>0$ such that $\int_{B_{1}} \frac{g(u(t)) \psi_{\epsilon}}{|x|^{2}}, \int_{B_{1}} \frac{u(t) \psi_{\epsilon}}{|x|^{2}} \leq M$ for all $t \geq 0$, or

2. $\int_{B_{1}} \frac{u(t) \psi_{e}}{|x|^{2}} \stackrel{t \rightarrow+\infty}{\longrightarrow}+\infty$.

Let us suppose that the second case holds. Then, by Jensen's inequality, we have for $t$ large enough :

$$
\frac{d}{d t} \int_{B_{1}} \frac{u(t) \psi_{\epsilon}}{|x|^{2}} \geq \frac{1}{2} \int_{B_{1}} \frac{g(u(t)) \psi_{\epsilon}}{|x|^{2}} \geq \frac{1}{2} g\left(\int_{B_{1}} \frac{u(t) \psi_{\epsilon}}{|x|^{2}}\right)
$$

Hence,

$$
\int_{0}^{f(t)} \frac{d s}{g(s)} \geq \frac{1}{2} t+C \text { where } f(t)=\int_{B_{1}} \frac{\underline{u}(t) \psi_{\epsilon}}{|x|^{2}}
$$

which contradicts (B7). And $u(t)=S(t) 0$ blows up in finite time.

Finally, suppose that the first case occurs. And let $\zeta$ denote the unique solution of the following problem :

$$
\left\{\begin{aligned}
-\left(|x|^{2}\right) \Delta \zeta & =1 \text { in } B_{1} \\
\zeta & \equiv 0 \text { in } \partial B_{1}
\end{aligned}\right.
$$

For $N \geq 3$, it is easy to prove that $\zeta \in W^{2, p}\left(B_{1}\right)$ for all $p<\frac{N}{2}$ which by Hardy's inequality and by Sobolev's embedding implies that $\zeta \in$ $L^{2}\left(\frac{d x}{|x|^{2}}\right) \cap H_{0}^{1}\left(B_{1}\right)$. Hence, there exists $\left\{\zeta_{n}\right\}_{n \in \mathbb{N}} \subset \mathcal{C}_{0}^{\infty}\left(B_{1}\right)$ such that :

$$
\Delta \zeta_{n} \stackrel{L^{1}}{\longrightarrow} \Delta \zeta \quad \text { and } \quad \zeta_{n} \stackrel{L^{2}\left(\frac{d x}{|x|^{2}}\right)}{\longrightarrow} \zeta
$$


Some results about blow-up and global...

339

Choosing $\phi:=\zeta_{n}$ in (3.5) and integrating in $[t, t+1]$, we have :

$$
\begin{aligned}
{\left[\int_{B_{1}} \frac{u(s) \zeta_{n}}{|x|^{2}}\right]_{t}^{t+1} } & +\int_{t}^{t+1} d s \int_{B_{1}} u(s)\left(-\Delta \zeta_{n}\right) \\
& =\int_{t}^{t+1} d s \int_{B_{1}} \frac{g(u(s)) \zeta_{n}}{|x|^{2}}
\end{aligned}
$$

Passing to the limit $n \rightarrow \infty$, we obtain by (3.6) :

$$
\int_{B_{1}} \frac{u(s) \zeta_{n}}{|x|^{2}} \stackrel{n \rightarrow \infty}{\longrightarrow} \int_{B_{1}} \frac{u(s) \zeta}{|x|^{2}}
$$

Moreover, by Lebesgue theorem and by (3.6) :

$$
\int_{t}^{t+1} d s \int_{B_{1}} \frac{g(u(s)) \zeta_{n}}{|x|^{2}} \stackrel{n \rightarrow \infty}{\longrightarrow} \int_{t}^{t+1} d s \int_{B_{1}} \frac{g(u(s)) \zeta}{|x|^{2}}
$$

and

$$
\int_{t}^{t+1} d s \int_{B_{1}} u(s)\left(-\Delta \zeta_{n}\right) \stackrel{n \rightarrow \infty}{\longrightarrow} \int_{t}^{t+1} d s \int_{B_{1}} u(s)(-\Delta \zeta)
$$

Therefore,

$$
\left[\int_{B_{1}} \frac{u(s) \zeta}{|x|^{2}}\right]_{t}^{t+1}+\int_{t}^{t+1} d s \int_{B_{1}} u(s)(-\Delta \zeta)=\int_{t}^{t+1} d s \int_{B_{1}} \frac{g(u(s)) \zeta}{|x|^{2}} .
$$

Now, since $u_{t} \geq 0$,

$$
\begin{aligned}
\int_{B_{1}} \frac{u(t)}{|x|^{2}} & \leq \int_{t}^{t+1} d s \int_{B_{1}} \frac{u(s) \zeta}{|x|^{2}}=\int_{t}^{t+1} d s \int_{B_{1}} u(s)(-\Delta \zeta) \\
& =\int_{t}^{t+1} d s \int_{B_{1}} \frac{g(u(s)) \zeta}{|x|^{2}}-\left[\int_{B_{1}} \frac{u(s) \zeta}{|x|^{2}}\right]_{t}^{t+1} \\
& \leq \int_{B_{1}} \frac{g(u(t+1)) \zeta}{|x|^{2}} \leq M
\end{aligned}
$$

Therefore, by monotone convergence, there exists $w \in L^{1}\left(\frac{d x}{|x|^{2}}\right)$ such that $u(t) \stackrel{t \rightarrow+\infty}{\longrightarrow} w$ in $L^{1}\left(\frac{d x}{|x|^{2}}\right)$. It implies that for all $\phi \in C_{0}^{2}\left(\bar{B}_{1}\right)$ :

$$
\begin{aligned}
& \int_{B_{1}} \frac{u(t) \phi}{|x|^{2}} \stackrel{t \rightarrow+\infty}{\longrightarrow} \int_{B_{1}} \frac{w \phi}{|x|^{2}}, \int_{t}^{t+1} d s \int_{B_{1}} u(s)(-\Delta \phi) \stackrel{t \rightarrow \infty}{\longrightarrow} \int_{B_{1}} w(-\Delta \phi) \text { and } \\
& \int_{t}^{t+1} d s \int_{B_{1}} \frac{g(u(s)) \phi}{|x|^{2}} \stackrel{t \rightarrow \infty}{\longrightarrow} \int_{B_{1}} \frac{g(w) \phi}{|x|^{2}}
\end{aligned}
$$


(For this, note that $\int_{t}^{t+1} d s \int_{B_{1}} \frac{g(u(s)) \phi}{|x| 2 \mid} \leq 2 \int_{B_{1}} \frac{w \phi}{|x|^{2}}<+\infty$ ). Therefore, for all $\phi \in C_{0}^{2}\left(\bar{B}_{1}\right)$ :

$$
-\int_{B_{1}} w \Delta \phi=\int_{B_{1}} \frac{g(w) \phi}{|x|^{2}}
$$

which contradicts the nonexistence of weak stationary solutions to $\left(P_{t}\right)$. This completes the proof of Theorem 3.3 .

Remarks. Consider $g \in C^{1}$ convex, increasing function satisfying $\lim _{s \rightarrow 0^{+}} \frac{g(s)}{s}>\left(\frac{N-2}{2}\right)^{2},(\mathrm{~B} 7)$ and for all $s \geq 0, \frac{s g(s)}{2} \geq G(s)=$ $\int_{0}^{s} g(t) d t$. Then, we can apply the previous method. Precisely, for all $u_{0}>0, u(\cdot)=S(\cdot) u_{0}$ blows up in finite time in $L^{2}\left(\frac{d x}{|x|^{2}}\right)$.

It suffices to modify the proof of Theorem 3.3 as follows :

1. 0 is replaced by $\epsilon \phi_{\epsilon}$ which is a subsolution of $(P)$ and $\epsilon \phi_{\epsilon}<u_{0}$, for $\epsilon$ small enough.

2. The nonexistence of stationary solutions of $\left(P_{t}\right)$ is provided by the results from [7].

\section{Global existence of solutions to $\left(P_{t}\right)$ and convergence to a stationary solution}

\subsection{Main results}

In this section, we give two examples of global existence of solutions to $\left(P_{t}\right)$ which converge to a stationary solution when $t \rightarrow \infty$. In each case, we obtain an exponential control of the convergence either in $L^{\infty}$ or in $H_{0}^{1}\left(B_{1}\right)$. Here, it is worth to underline that the convergence to a stationary solution is related to the uniqueness of the solution to $(P)$. First, we prove the following :

Theorem 4.1. Assume that $N \geq 2$ and the following hypothesis :

(G1) $\lim _{s \rightarrow 0^{+}} \frac{g(s)}{s}=\lambda<0$, 
(G2) There exists $\epsilon>0$, such that $|g(s)-\lambda s| \leq C|s|^{1+\epsilon}$.

Then, for $u_{0}$ stuch that $\left\|u_{0}\right\|_{L^{\infty}}$ small enough, $u(\cdot)=S(\cdot) u_{0}$ is global and there exists $C>0$ such that $\|u(t)\|_{L^{\infty}} \leq C e^{\lambda t}$ for all $t \geq 0$.

In the second part of the section, we prove the following theorem :

Theorem 4.2. Assume that $N \geq 3$ and $g$ satisfies the following assumptions :

(G3) $s \rightarrow \frac{g(s)}{s}$ is continuous and strictly decreasing,

(G4) $\stackrel{g(s)}{s} \stackrel{s \rightarrow+\infty}{\longrightarrow}-\infty$,

(G5) $\stackrel{g(s)}{s} \stackrel{s \rightarrow 0^{+}}{\longrightarrow} \lambda>\left(\frac{N-2}{2}\right)^{2}$,

Then, for any $u_{0}$ such that $0<u_{0} \leq f^{-1}(0)$ and $u_{0} \not \equiv f^{-1}(0)$, with $f(s):=\frac{g(s)}{s}, u(t)=S(t) u_{0}$ is global and converges to the unique nontrivial solution of $(P), w_{\lambda}$, when $t \rightarrow \infty$. Moreover, if we suppose, in addition, that $-g$ is strictly convex, there exists $K>0$ such that $\left\|u(t)-w_{\lambda}\right\|_{H_{0}^{1}\left(B_{1}\right)} \leq C e^{-K t}$ for all $t \geq 0$.

We start by proving a proposition which provides the heat kernel of $-|x|^{2} \Delta$ :

Proposition 4.3. Consider $u=T(t) u_{0} \in L^{\infty}\left(B_{1}\right) \cap L^{2}\left(\frac{d x}{|x|^{2}}\right)$ solution of

$$
\left\{\begin{aligned}
u_{t}-|x|^{2} \Delta u & =\lambda u \text { in } B_{1} \\
u(t, x) & =0 \text { in } \mathbb{R}^{+} \times \partial B_{1}, u(0, x)=u_{0}
\end{aligned}\right.
$$

where $u_{0}$ is radial. Then, $u(t)$ is radial and if $v(t, s):=u(t, x)$ with $s=-\ln |x|$ and $\lambda_{N}=\left(\frac{N-2}{2}\right)^{2}$, then,

$$
v(t, s)=\frac{e^{\frac{N-2}{2} s-\left(\lambda_{N}-\lambda\right) t-\frac{|s|^{2}}{4 t}}}{(4 \pi t)^{\frac{1}{2}}} * v(0, s) .
$$

Proof. First, we remark that the radial symmetry of $u$ follows from the uniqueness of the solution to $\left(P_{t}\right)$. Then, to compute the heat kernel of 
$-|x|^{2} \Delta$, we use a method from [7]. Indeed, put $w(t, s):=e^{-\frac{N-2}{2} s} v(t, s)$. We show that $w$ satisfies :

$$
\left(P_{w}\right)\left\{\begin{aligned}
w_{t}-w_{s s} & =\left(\lambda-\lambda_{N}\right) w \text { in } \mathbb{R}^{+} \times(0,+\infty) \\
w(t, 0) & =0, w(t, s) \stackrel{s \rightarrow+\infty}{\longrightarrow} 0, w(0, s)=v(0, s) e^{-\frac{N-2}{2} s}
\end{aligned}\right.
$$

Taking $w(t,-s)=-w(t, s)$ for all $s \geq 0$, we have that $\left(P_{w}\right)$ is satisfied in $\mathbb{R}^{+} \times \mathbb{R}$. And we can apply Fourier transform. Indeed, for $N \geq 2$, $w(t, \cdot)$ belongs to $L^{2}(R)$ (for $N>2$, it is obvious since $v \in L^{\infty}$ and for $N=2$, it suffices to remark that $\left.\int_{B_{1}} \mid \frac{|u|^{2}}{|x|^{2}}<\infty \Rightarrow \int_{0}^{+\infty} w^{2} d s<\infty\right)$.

A simple computation shows that $\hat{w}(t, x)=\hat{w}_{0} e^{-\left(|x|^{2}+\left(\lambda_{N}-\lambda\right) t\right)}$. Using inverse Fourier transform, one has :

$$
w(t, s)=w_{0} * \frac{e^{-\left(\lambda_{N}-\lambda\right) t-\frac{|s|^{2}}{4 t}}}{(4 \pi t)^{\frac{1}{2}}} \text { and } v(t, s)=v_{0} * \frac{e^{\frac{N-2}{2} s-\left(\lambda_{N}-\lambda\right) t-\frac{|s|^{2}}{4 t}}}{(4 \pi t)^{\frac{1}{2}}}
$$

This completes the proof of Proposition 4.3.

Now, we are ready to prove Theorem 4.1.

Proof of Theorem 4.1. Here, we apply a method from [4]. First, remark that by the maximum principle, it suffices to prove Theorem 4.1 when $u_{0}$ is radially symmetric. Then, by Proposition 4.3, $T(t) u_{0}$ is radially symmetric and

$$
\begin{aligned}
\left\|T(t) u_{0}\right\|_{L^{\infty}} & =\left\|v_{0} * \frac{e^{\frac{N-2}{2} s-\left(\lambda_{N}-\lambda\right) t-\frac{|s|^{2}}{4 t}}}{(4 \pi t)^{\frac{1}{2}}}\right\|_{L^{\infty}} \\
& \leq\left\|v_{0}\right\|_{L^{\infty}}\left\|\frac{e^{\frac{N-2}{2} s-\left(\lambda_{N}-\lambda\right) t-\frac{|s|^{2}}{4 t}}}{(4 \pi t)^{\frac{1}{2}}}\right\|_{L^{1}}
\end{aligned}
$$

Now, using Laplace transform $f(y):=\int_{-\infty}^{+\infty} e^{y x-\frac{|x|^{2}}{4 t}} d x$, we show that $\left\|e^{\frac{N-2}{2} s-\frac{s^{2}}{42}}\right\|_{L^{1}}=$ $e^{t \lambda_{N}}(4 \pi t)^{\frac{1}{2}}$. Therefore, by (4.1), $\left\|T(t) u_{0}\right\|_{L^{\infty}} \leq e^{\lambda t}\left\|u_{0}\right\|_{L^{\infty}}$. Now, we apply a method from [4]. First, we define $\theta(\cdot)$ such that $\theta(x)=$ $\frac{C}{\epsilon|\lambda|} x^{1+\varepsilon}-x$ with $C$ defined in (G2) and $\delta>0$ satisfy

$$
\min \theta(x)+\delta<0, \Theta(\delta)+\delta \geq 0 \text { and } \Theta^{\prime}(\delta) \leq 0
$$


Let us choose $u_{0} \in L^{\infty} \cap L^{2}\left(\frac{d x}{|x|^{2}}\right)$ such that $\left\|u_{0}\right\|_{L^{\infty}} \leq \delta$. Then, $u=$ $S(t) u_{0}$ satisfies :

$$
\begin{aligned}
\|u(t)\|_{L^{\infty}} \leq\left\|T(t) u_{0}\right\|_{L^{\infty}} & +C \int_{0}^{t} e^{\lambda(t-s)}\|u(s)\|_{L^{\infty}}^{1+\epsilon} \leq e^{\lambda t} \delta \\
& +C e^{\lambda t} \int_{0}^{t} e^{\epsilon \lambda s}\left(e^{-\lambda s}\|u(s)\|_{L^{\infty}}\right)^{1+\epsilon} d s
\end{aligned}
$$

Putting $\phi(t)=\sup _{[0, t]} e^{-\lambda s}\|u(s)\|_{L^{\infty}}$ which is an increasing function, we have :

$$
\phi(t) \leq \delta+C \int_{0}^{t} \phi^{1+\varepsilon}(s) e^{\epsilon \lambda s} d s \leq \delta+\frac{C}{\epsilon|\lambda|} \phi(t)^{1+\epsilon} .
$$

If $\mu=\inf \{x>0 / \Theta(x)+\delta \leq 0\} \geq \delta$, it is easy to prove that $\phi(t) \leq \mu$ for all $t \in[0, T[$, where $T$ is defined in Proposition 2.3. Moreover, $\|u(t)\|_{L^{\infty}} \leq e^{\lambda t} \mu$, which implies that $u$ is global and $T=\infty$. This completes the proof of Theorem 4.1.

\section{Remarks.}

1. If $p \in] 1,+\infty\left[\right.$, the function $g: s \rightarrow s^{p}$ satisfies the hypothesis of Theorem 4.1. Therefore, Theorems 3.1 and 4.1 show that the behaviour of the solution of $\left(P_{t}\right)$ depends on the initial data.

2. It is worth noticing that for $N=2$, we obtain almost a complete description of the behaviour of solutions of $\left(P_{t}\right)$. Precisely, $\lambda_{N}=$ $\left(\frac{N-2}{2}\right)^{2}=0$ is the "blow-up critical parameter" (see [13]) which means that for $\lambda<\lambda_{N}$, there exists global solutions of $\left(P_{t}\right)$ for small initial data and if $\lambda>\lambda_{N}$ then for all $u_{0} \not \equiv 0, u(t)=S(t) u_{0}$ blows up in finite time. However, we do not know what happens in the case $\lambda=\lambda_{N}$. Moreover, since the heat kernel of $-|x|^{2} \Delta$ does not vanish at the boundary, we cannot apply a method due to Fujita (see [9]) which would furnish the answer. For $N \geq 3$, we suspect that $\lambda_{N}$ still remains the critical blow-up parameter.

Now, we give the proof of Theorem 4.2 . 


\section{Proof of Theorem 4.2.}

Since there is a unique nontrivial solution to $(P)$, it suffices to prove Theorem 4.2 when $u_{0}$ is radially decreasing. In this case, $S(t) u_{0}$ is also radially decreasing. Indeed, choosing $\epsilon \in] 0,1[$, we remark that $u(t, \epsilon x):=u_{\varepsilon}(t)$ is solution to

$$
\left(P_{\epsilon, t}\right)\left\{\begin{array}{l}
u_{t}-|x|^{2} \Delta u=g(u) \text { in } \mathbb{R}^{+} \times B_{\frac{1}{\epsilon}} \\
u(t, x)=0 \text { in } \mathbb{R}^{+} \times \partial B_{\frac{1}{\epsilon}}, u(0, x)=u_{0}(\epsilon x)
\end{array}\right.
$$

Since $u_{0}(\epsilon x) \geq u_{0}(x)$, by the maximum principle, for any $\left.\epsilon \in\right] 0,1[$, $u_{\ell}(t) \geq S(t) u_{0}$ which proves that $S(t) u_{0}$ is radially decreasing.

Now, as above we prove that :

$$
. \quad \frac{d}{d t} \int_{B_{1}} \frac{|u(t)|^{2}}{|x|^{2}}=-\int_{B_{1}}|\nabla u(t)|^{2}+\int_{B_{1}} \frac{g(u(t)) u(t)}{|x|^{2}}
$$

Moreover, $E(u(t))=\frac{1}{2} \int_{B_{1}}|\nabla u(t)|^{2}-\int_{B_{1}} \frac{G(u(t))}{|x|^{2}}$ satisfies

$$
\frac{d}{d t} E(u(t))<0 \text { and } E(u(t))<E\left(u_{0}\right)
$$

Futhermore, multiplying the equation in $\left(\dot{P}_{t}\right)$ by $\frac{\left(u-f^{-1}(0)\right)^{+}}{|x|^{2}}$ we obtain :

$$
\begin{aligned}
\frac{d}{d t} \int_{B_{1}} \frac{\left(\left(u(t)-f^{-1}(0)\right)^{+}\right)^{2}}{|x|^{2}} & +\int_{B_{1}}\left|\nabla\left(u(t)-f^{-1}(0)\right)^{+}\right|^{2} \\
& =\int_{B_{1}} \frac{\left((\lambda-f(u(t)))\left(u-f^{-1}(0)\right)^{+}\right)^{2}}{|x|^{2}} \leq 0
\end{aligned}
$$

which implies that for all $t \geq 0, u(t) \leq f^{-1}(0)$ and therefore $\cup_{t \geq 0}\{u(t)\}$ is uniformly bounded in $L^{\infty}\left(B_{1}\right)$. By (4.2), for $N \geq 3$, it follows that :

$$
\int_{B_{1}} \frac{|\nabla u(t)|^{2}}{2} \leq E\left(u_{0}\right)-G\left(f^{-1}(0)\right) \int_{B_{1}} \frac{1}{|x|^{2}} d x \leq C
$$

Therefore, $\cup_{t \geq 0}\{u(t)\}$ is bounded in $L^{\infty}\left(B_{1}\right) \cap H_{0}^{1}\left(B_{1}\right)$. Then, for any sequence $\left\{t_{n}\right\}_{n \in \mathbb{N}}$ such that $t_{n} \rightarrow+\infty$, there is $w \in L^{\infty}\left(B_{1}\right) \cap H_{0}^{1}\left(B_{1}\right)$ (depending a priori on $\left\{t_{n}\right\}_{n \in N}$ ) satisfying

$$
u\left(t_{n}\right) \stackrel{n \rightarrow \infty}{\rightarrow} w \text { in } H_{0}^{1}\left(B_{1}\right), u\left(t_{n}\right) \stackrel{n \rightarrow \infty}{\rightarrow} w \text { in } L^{2}\left(\frac{d x}{|x|^{2}}\right)
$$


and

$$
G\left(u\left(t_{n}\right) \stackrel{n \rightarrow \infty}{\rightarrow} G(w) \text { in } L^{1}\left(\frac{d x}{|x|^{2}}\right) .\right.
$$

For this, notice that on one hand

$$
\int_{B_{1}} \frac{\left|u\left(t_{n}\right)-w\right|^{2}}{|x|^{2}} \leq\left(\int_{B_{1}} w\left(t_{n}\right)-\left.w\right|^{p^{\prime}}\right)^{\frac{1}{p^{\prime}}}\left(\int_{B_{1}} \frac{1}{|x|^{2 p}}\right)^{\frac{1}{p}}
$$

where $p<\frac{N}{2}$ and $\frac{1}{p}+\frac{1}{\vec{p}}=1$. On the other hand, since $\cup_{t \geq 0}\{u(t)\}$ and $w$ are uniformly bounded in $L^{\infty}$,

$$
\int_{B_{1}} \frac{\mid G\left(u\left(t_{n}\right)-\left.G(w)\right|^{2}\right.}{|x|^{2}} \leq C \int_{B_{1}} \frac{\left|u\left(t_{n}\right)-w\right|^{2}}{|x|^{2}}
$$

Let us show that

$$
u\left(t_{n}\right) \stackrel{H_{0}^{1}\left(B_{1}\right)}{\longrightarrow} w \text { when } n \rightarrow \infty
$$

For this, it suffices to prove that $\int_{B_{1}}\left|\nabla u\left(t_{n}\right)\right|^{2} \stackrel{n \rightarrow \infty}{\longrightarrow} \int_{B_{1}}|\nabla w|^{2}$. Let us prove that $\int_{B_{1}}\left|\nabla u\left(t_{n}\right)\right|^{2}$ does not concentrate in $x=0$. First, for any $\delta<1$, multiplying the equation in $\left(P_{t}\right)$ by $\frac{u(t)}{|x|^{2}}$ in $B_{\delta}$, we have :

$\frac{d}{d t} \int_{|x| \leq \delta} \frac{|u|^{2}}{|x|^{2}}+\int_{|x| \leq \delta}|\nabla u(t)|^{2}-\int_{|x|=\delta} \frac{\partial u(t)}{\overline{\partial n}} u(t) d s=\int_{|x| \leq \delta} \frac{g(u(t)) u(t)}{|x|^{2}}$

Since $u(t)$ is radially decreasing,

$$
\frac{d}{d t} \int_{|x| \leq \delta} \frac{|u|^{2}}{|x|^{2}}+\int_{|x| \leq \delta}|\nabla u(t)|^{2} \leq \int_{|x| \leq \delta} \frac{g(u(t)) u(t)}{|x|^{2}}
$$

Integrating (4.3) in $[t, t+1]$, we obtain :

$$
\left[\int_{|x| \leq \delta} \frac{u(s)^{2}}{|x|^{2}}\right]_{t}^{t+1}+\int_{t}^{t+1} d s \int_{|x| \leq \delta}|\nabla u(s)|^{2} \leq C \int_{|x| \leq \delta} \frac{1}{|x|^{2}}
$$

where $C$ is independent of $t$. Then, for all $\epsilon>0$, there is $\delta(\epsilon)>0$ small enough such that for all $\delta \leq \delta(\epsilon)$, we have :

$$
0 \leq \int_{t}^{t+1} \int_{|x| \leq \delta}|\nabla u(t)|^{2} \leq \epsilon
$$


To conclude the proof, suppose that $\int_{B_{1}}|\nabla w|^{2}<\lim _{n \rightarrow \infty} \int_{B_{1}}\left|\nabla u\left(t_{n}\right)\right|^{2}$. Then, by (4.2) $E(w)<E_{\infty}=\lim _{t \rightarrow \infty} E(u(t))$. However, by (4.4), it is easy to prove that

$$
\int_{t}^{t+1} d s \int_{B_{1}}\left|\nabla u\left(t_{n}+\tau\right)\right|^{2} \stackrel{n \rightarrow \infty}{\longrightarrow} \int_{t}^{t+1} \int_{B_{1}}|\nabla(S(\tau) w)|^{2}
$$

Indeed, by the boundedness of $\{u(t)\}_{t \geq 0}$ in $L^{\infty} \cap H_{0}^{1}\left(B_{1}\right)$,

$$
\begin{aligned}
\left\||x|^{2} \Delta\left(S(t+\tau) u_{0}\right)\right\|_{L^{2}\left(\frac{d x}{|x|^{2}}\right)} & \leq\left\||x|^{2} \Delta\left(T(t) u_{0}\right)\right\|_{L^{2}\left(\frac{d x}{|x|^{2}}\right)} \\
& +\int_{t}^{t+\tau}\left\||x|^{2} \Delta T(t+\tau-s) g(u(s))\right\|_{L^{2}\left(\frac{d x}{|x|^{2}}\right)} d s
\end{aligned}
$$

from which it follows :

$$
\begin{aligned}
\left\||x|^{2} \Delta S(t+\tau) u_{0}\right\|_{L^{2}\left(\frac{d x}{|x|^{2}}\right)} & \leq \frac{C}{\tau}\|u(t)\|_{L^{\infty}} \\
& +C \int_{t}^{t+\tau} \frac{d s}{(t+\tau-s)^{\frac{1}{2}} s^{\frac{1}{2}}}\left\|u_{0}\right\|_{L^{\infty}} \leq \frac{G}{\tau}
\end{aligned}
$$

(for this, using a method from [4] Lemma 3.10, we prove that

$$
\left\||x|^{2} \Delta T(t) u_{0}\right\|_{L^{2}\left(\frac{d x}{|x|^{2}}\right)} \leq \frac{1}{t^{\frac{1}{2}}}\left\|u_{0}\right\|_{H_{0}^{1}\left(B_{1}\right)}
$$

and

$$
\left\|T(t) u_{0}\right\|_{H_{0}^{1}\left(B_{1}\right)} \leq t^{-\frac{1}{2}}\left\|u_{0}\right\|_{L^{2}\left(\frac{d x}{|x|^{2}}\right)} \leq C t^{-\frac{1}{2}}\left\|u_{0}\right\|_{L^{\infty}}
$$

for $N \geq 3$ )

Finally, (4.4) and the compactness of the embedding $H^{2}(\delta \leq|x| \leq$ 1) $\hookrightarrow H^{1}(\delta \leq|x| \leq 1)$ imply (4.5). Now, using that $E(\cdot)$ is decreasing, we have :

$$
\int_{t}^{t+1} E(S(\tau) w) d \tau \leq E(w)<E_{\infty}
$$

which contradicts (4.5). Thus,

$$
\int_{B_{1}}\left|\nabla u\left(t_{n}\right)\right|^{2} \stackrel{n \rightarrow \infty}{\longrightarrow} \int_{B_{1}}|\nabla w|^{2}
$$


and for any

$$
t \geq 0 E(S(t)(w))=E(w)=E_{\infty} .
$$

This implies that $w$ is a stationary solution of $\left(P_{t} \cdot u_{0}\right)$ and either $w \equiv 0$, or $w \equiv w_{\lambda}$ which is the nontrivial solution of $(P)$.

Now, let us prove that $u(t) \stackrel{t \rightarrow \infty}{\longrightarrow} w$ in $L^{\infty}\left(B_{1}\right)$. By a bootstrap argument (see [12]), it is easy to prove that for any $\delta>0$,

$$
\|u(t)-w\|_{L^{\infty}(|x| \geq \delta)} \stackrel{t \rightarrow \infty}{\longrightarrow} 0
$$

We consider $u_{0}:=\epsilon \psi_{\epsilon}$ which satisfies

$$
|x|^{2} \Delta u_{0}+g\left(u_{0}\right) \geq 0 \text { if } \epsilon \text { is small enough }
$$

We recall that $\psi_{c}$ is the eigenfunction of $-\left(|x|^{2}+|\epsilon|^{2}\right) \Delta$ defined in the proof of Theorem 3.1. Note that by (G5) and (G4), (4.7) is satisfied for $\epsilon$ small enough. Then, $u_{0}$ is a strict subsolution of $(P)$ and as above, it implies that $\frac{d}{d t} S(t) u_{0} \geq 0$ for all $t \geq 0$. Hence, $u(\cdot)$ is increasing. Hence, $w=v_{\lambda}$. Then, by Dini's theorem and (4.6), we have for all $\delta>0$ :

$$
\left\|u(t)-w_{\lambda}\right\|_{L^{\infty}(|x| \geq \delta)} \stackrel{t \rightarrow \infty}{\longrightarrow} 0
$$

Moreover, from [8] we know that $w_{\lambda}(0)=f^{-1}(0)$. Therefore, since $\{u(t)\}_{t \geq 0} \cup\left\{w_{\lambda}\right\}$ are radially decreasing

$$
\begin{aligned}
\limsup _{t \rightarrow \infty}\left\|w_{\lambda}-u(t)\right\|_{L^{\infty}\left(B_{1}\right)} & \leq \limsup _{t \rightarrow \infty}\left\|u(t)-w_{\lambda}\right\|_{L^{\infty}(|x| \leq \delta)} \\
& +\limsup _{t \rightarrow \infty}\left\|u(t)-w_{\lambda}\right\|_{L^{\infty}(|x| \geq \delta)} \\
& =\limsup _{t \rightarrow \infty}\left\|u(t)-w_{\lambda}\right\|_{L^{\infty}(|x| \leq \delta) \leq f^{-1}(0)} \\
& -\lim _{t \rightarrow \infty} \lim _{|x| \rightarrow 0} u(t, x)
\end{aligned}
$$

Thus, suppose that $c_{\lambda}:=\lim _{t \rightarrow \infty} \lim _{|x| \rightarrow 0} u(t, x)<f^{-1}(0)$. Then, since $u(t, \cdot)$ is radially decreasing, for any $x_{\delta}$ such that $|x|=\delta>0, w_{\lambda}\left(x_{\delta}\right)=$ $\lim _{t \rightarrow \infty} u\left(t, x_{\delta}\right) \leq c_{\lambda}$. This contradicts that $w_{\lambda}$ is continuous.

Now, considering any $u_{0}$ such that $0<u_{0} \leq f^{-1}(0)$ and $u_{0} \neq f^{-1}(0)$, there exists $\epsilon>0$ small enough such that $\epsilon \psi_{\epsilon}<u_{0}$. It implies that

$$
S(t)\left(\epsilon \psi_{\epsilon}\right)<S(t)\left(u_{0}\right) \leq f^{-1}(0) \text { and }\left\|S(t) u_{0}-w_{\lambda}\right\|_{L^{\infty}\left(B_{1}\right)} \stackrel{t \rightarrow \infty}{\longrightarrow} 0(4.9)
$$


To conclude the proof of Theorem 4.2, let us prove that if we suppose, in addition, that $-g$ is strictly convex, then, there exists $K>0$ such that $\left\|u(t)-w_{\lambda}\right\|_{H_{0}^{\mathrm{z}}\left(B_{1}\right)} \leq C e^{-K^{t}}$ for all $t \geq 0$. First, note that

$$
\begin{aligned}
\frac{d}{d t} \int_{B_{1}} \frac{\left|w_{\lambda}-u(t)\right|^{2}}{|x|^{2}} & +\int_{B_{1}}\left|\nabla\left(w_{\lambda}-u(t)\right)\right|^{2}- \\
& =\int_{B_{1}} \frac{\left(g\left(w_{\lambda}\right)-g(u(t))\right)\left(w_{\lambda}-u(t)\right)-}{|x|^{2}}
\end{aligned}
$$

By (4.9), for $t$ large enough, we have :

$$
\frac{d}{d t} \int_{B_{1}} \frac{\left|w_{\lambda}-u(t)\right|^{2}}{|x|^{2}}+\frac{\lambda_{1}}{2}\left(-\Delta-\frac{g^{\prime}\left(w_{\lambda}\right)}{|x|^{2}}\right) \int_{B_{1}} \frac{\left(w_{\lambda}-u(t)\right)^{2}}{|x|^{2}} \leq 0
$$

where $\frac{\lambda_{1}}{2}\left(-\Delta-\frac{g^{\prime}\left(w_{\lambda}\right)}{\partial x}\right)$ is the first eigenvalue of $\left(-\Delta-\frac{g^{\prime}\left(w_{\lambda}\right)}{|\lambda|^{2}}\right)$ in $H_{0}^{1}\left(B_{1}\right)$. Then, the strict convexity of $-g$ implies (see [8]) :

$$
\lambda_{1}\left(-\Delta-\frac{g^{\prime}\left(w_{\lambda}\right)}{|x|^{2}}\right)>\lambda_{1}\left(-\Delta-\frac{1}{|x|^{2}} \frac{g\left(w_{\lambda}\right)}{w_{\lambda}}\right)=0
$$

Thus, from $(4.10)$, it is easy to prove that:

$$
\int_{B_{1}} \frac{\left|w_{\lambda}-u(t)\right|^{2}}{|x|^{2}} \leq C e^{-\frac{\lambda_{1} t}{2}}
$$

Using (4.10) and putting $K=\frac{\lambda_{1}}{2}$, we have for all $t$ :

$$
\int_{B_{1}}\left|\nabla\left(w_{\lambda}-u(t)\right)\right|^{2} \leq C e^{-K t}
$$

This completes the proof of Theorem 4.2.

\section{Remarks.}

1. If $g(s):=\lambda s-|s|^{p-1} s$ where $\lambda>\left(\frac{N-2}{2}\right)^{2}$ and $p>1$, then, $g$ satisfies the assumptions of Theorem 4.2 . 
2. Suppose that $-g$ is strictly convex and satisfies the assumptions (G3) to (G5). Then, taking $\delta>0,(4.11)$ and a bootstrap argument show that for all $t \geq 0$ :

$$
\left\|u(t)-w_{\lambda}\right\|_{L^{\infty}(|x| \geq \delta)} \leq C(\delta) e^{-K t} .
$$

However, we don't know if that remains valid for $\delta=0$.

3. The assumption (G5) and the second part of Assumption (G4) suffice to prevent that $u(t)=S(t) u_{0}$ converges to 0 in $L^{\infty}\left(B_{1}\right)$ when $t \rightarrow \infty$ and when $u_{0} \neq 0$.

Indeed, suppose that $\left\|S(t) u_{0}\right\|_{L^{\infty}\left(B_{1}\right)} \stackrel{t \rightarrow \infty}{\longrightarrow} 0$. Then, adapting a method from [14], we consider $\epsilon$ small enough such that $\lambda_{\epsilon}^{1}<\lambda$. Then, multiplying the equation in $\left(P_{t}\right)$ by $\psi_{\varepsilon}$ :

$$
\frac{d}{d t} \int_{B_{1}} \frac{u(t) \psi_{\epsilon}}{|x|^{2}}+\lambda_{\epsilon}^{1} \int_{B_{\mathfrak{l}}} \frac{u(t) \psi_{\epsilon}}{|x|^{2}+|\epsilon|^{2}}=\int_{B_{1}} \frac{g(u(t)) \psi_{\epsilon}}{|x|^{2}}
$$

from which it follows for $t$ large enough :

$$
\begin{aligned}
\frac{d}{d t} \int_{B_{1}} \frac{u(t) \psi_{\epsilon}}{|x|^{2}} & \geq-\lambda_{\epsilon}^{1} \int_{B_{1}} \frac{u(t) \psi_{\epsilon}}{|x|^{2}}+\int_{B_{1}} \frac{g(u(t)) \psi_{\epsilon}}{|x|^{2}} \\
& \geq \frac{1}{2}\left(g^{\prime}(0)-\lambda_{\epsilon}^{1}\right) \int_{B_{1}} \frac{u(t) \psi_{\epsilon}}{|x|^{2}}
\end{aligned}
$$

Moreover, assumption (G5) implies that for $\epsilon$ small enough, $g^{\prime}(0)=$

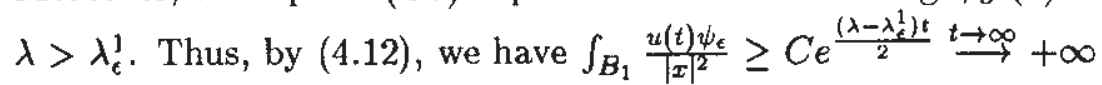
which contradicts the uniform boundedness of $\{u(t)\}_{t \geq 0}$.

4. In [8], the authors show the existence and the uniqueness of the solution, $u_{\epsilon}$, to the following pertubed problem :

$$
\left(P_{\varepsilon}\right)\left\{\begin{array}{l}
-|x|^{2} \Delta u=g(u)+\epsilon f(u) \text { in } B_{1} \\
u \in H_{0}^{1}\left(B_{1}\right), u \geq 0
\end{array}\right.
$$

where $g$ satisfies (G3) to (G5), $f$ is a positive function in $\mathbb{R}^{+}$and belongs to $C^{1}\left(\mathbb{R}^{+}\right)$such that $\lim _{s \rightarrow+\infty} f(s)+g(s)=-\infty$ and $\epsilon>0$ small enough. Moreover, $\lambda_{1}\left(-\Delta-\frac{\left(g^{\prime}\left(u_{\varepsilon}\right)+\epsilon f^{\prime}\left(u_{\varepsilon}\right)\right)}{|x|^{2}}\right)>0$. Then, Theorem 4.2 holds for $\left(P_{\epsilon}\right)$. 
Jacques Giacomoni

\section{References}

[1] H. Berestycki, Le nombre de solutions de certains problèmes semilinéaires elliptiques. J. Funct. Ana]. 40 (1981), 1-29.

[2] H. Berestycki and M.J. Esteban, Existence and bifuncation of solutions for an elliptic degenerate problem. J.D.E. 134, No. 1 (1997).

[3] H. Brezis, T. Cazenave, Y. Martel and A. Ramiandrisoa, Blow up for $u_{t}-\Delta u=g(u)$ revisited. Advances in Differential Equations 1 (1996), 76-91.

[4] T. Cazenave and A. Haraux, Introduction aux problèmes d'évolution semi-linéaires, mathématiques et applications. 1, Ellipses, Paris, 1990.

[5] T. Cazenave and P. L. Lions, Solutions globales d'équations de la chaleur semi-linéaires. Comm. P. D. E. 9 (1984), 955-978.

[6] M. Escobedo, M.A. Herrero and J.J.L. Velázquez, A nonlinear Fokker-Planck equation modelling the approach to thermal equilibrium in a homogeneous plasma. Preprint.

[7] M.J. Esteban and M. Ramaswamy, Nonexistence result for positive solutions of nonlinear elliptic degenerate problems. Nonlinear Analysis, Theory, Methods and Applications. 26, No. 4 (1996), 835-843.

[8] M.J. Esteban and J. Giacomoni, Existence of global branches of positive solutions for semilinear elliptic degenerate problems. Preprint.

[9] H: Fujita, On the blowing up of solutions of the Cauchy problem for $u_{t}=\Delta u+u^{1+\alpha}$. J. Fac. Sci. Univ. Tokyo. Section I.A. Math. 13 (1966), 109-124.

[10] B. Gidas and J. Spruck, A priori bounds for positive solutions of nonlinear elliptic equations. Commun. P. D. E. 6 (1981), 883-901.

[11] Y. Giga, A bound for global solutions of a semilinear heat equations. Commun. Math. Phys. 103 (1986), 415-421. 
[12] O. A. Ladyzhenkaya, V. A Solonnikov, N. N. Uralceva, Linear and quasilinear equations of parabolic type. Transl. Math. Monogr. 23 (1968), Providence, R.I. Am. Math. Soc. V. A.

[13] H. A. Levine, The role of critical exponents in blow up Theorems. SIAM Review. 32 No. 2 (1990), 262-288.

[14] M. A. Jendoubi, Exponential stability of positive solutions to some nonlinear heat equations. Preprint.

[15] K. Yosida, Functionnal Analysis. Springer (1978).

CEREMADE (URA.CNRS N 749),

Université de Paris-Dauphine,

75775 Paris Cedex 16, France

e-mail: giacomoeceremade. dauphine.fr

Recibido: 6 de Octubre de 1997

Revisado: 9 de Febrero de 1998 